\title{
Validity of over-the-counter finger stick glucose measurement devices in comparison with laboratory venous plasma glucose measurements on pregnant women with diabetes
}

\author{
S Muhandiram ${ }^{1}$, D H Suranimala ${ }^{1}$, N D Weerasekara², C Ratnayake ${ }^{1}$ \\ (Index words: validity, self-monitoring blood glucose devices, glucometer, diabetes mellitus, pregnancy)
}

\begin{abstract}
Introduction Self-monitoring of fasting, pre and postprandial blood glucose (SMBG) is an integral part of diabetic care. The validity and accuracy of different glucometer types vary significantly and at present, none of the local authorities carry out pre or post marketing evaluation of these devices in Sri Lanka. This study evaluated the accuracy and validity of 3 types of SMBG devices available over the counter, Device 1, Device 2 and Device 3 against a standard laboratory reference method.
\end{abstract}

Methods A random sample of 103 capillary and venous blood samples were analyzed from diabetic pregnant patients admitted to Teaching Hospital Peradeniya for Blood Sugar Series Testing. Haematocrit value was also obtained by microhaematocrit method. Analytical and clinical accuracy were determined.

Results The correlation coefficiencies were $0.856,0.880$ and 0.873 for Device 1, Device 2 and Device 3, respectively. Only Device 2 met the International Standardization Organization (ISO) performance standards for glucometers for glucose values $>100 \mathrm{mg} / \mathrm{dL}$ whereas none of them met the ISO criteria for values $<100 \mathrm{mg} /$ dL. In surveillance error grids, $87.8 \%, 93.3 \%$ and $81.6 \%$ of the data points were within non risk zone for Device 1 , Device 2 and Device 3 whereas $11.1 \%, 4.4 \%$ and $17.2 \%$ were within slightly lower risk hypoglycemia area. All types of glucometers were less reliable in detecting hypoglyacaemia.

Conclusions The over the counter SMBG monitoring systems are encountering certain problems in terms of accuracy and validity. Clinicians should exercise caution in making clinical decisions based on glucometer values for diabetic pregnant patients.

Ceylon Medical Journal 2018; 63: 180-185

DOI: http://doi.org/10.4038/cmj.v63i4.8769

\section{Introduction}

The prevalence of diabetes during pregnancy has been rising to epidemic proportions globally and particularly in developing countries like Sri Lanka [1]. Diabetes during pregnancy is a chronic illness that confer greater risk of maternal and fetal complications. Generally, some of the specific fetal, neonatal and maternal complications associated with uncontrolled diabetes include congenital malformations, macrosomia, spontaneous abortions, preeclampsia, caesarian sections, preterm labor, neonatal hypoglycemia and neonatal hyperbilirubinemia [2]. According to the American Diabetes association (ADA) standards on management of diabetes during pregnancy, two primary methods have been recommended to assess the effectiveness of the management plan of pregnant women with diabetes including self-monitoring of blood glucose by patients themselves (SMBG) and testing $\mathrm{HbA} 1 \mathrm{C}$ value [3]. Hence frequent self-monitoring of blood glucose value is indispensable for preventing hypoglycemia unawareness, determination of the most effective therapeutic modality for diabetic care, determination of prandial insulin doses, adjusting medical nutritional therapy and physical activities [4].

Despite SMBG devices being considered a fast, accurate, portable and cost effective method of blood glucose estimation, there are situations where SMBG measurements are not reliable owing to confounding nature of factors affecting the final reading [5,6,7]. Multiple variables affecting glucometer blood glucose estimations leading to errors and inaccuracies includes, but not limited to, strip factors (composition of the chemical strip, manufacturer variances, enzymes used in glucose oxidation and storage conditions) [8], physical factors (temperature, hypoxia, humidity), patient factors (hematocrit, severe hypo- or hyperglycemia or peripheral circulatory failure, elevated cholesterol (>13 $\mathrm{mmol} / \mathrm{l})$, interference from drugs

\footnotetext{
${ }^{1}$ Department of Obstetrics and Gynecology, Faculty of Medicine, University of Peradeniya, ${ }^{2}$ Teaching Hospital Peradeniya, Peradeniya, Sri Lanka.
}

Correspondence: CR e-mail: <chathura.ratnayake@gmail.com>. Received 29 September 2018 and revised version accepted 03 October 2018.

This is an open-access article distributed under the terms of the Creative Commons Attribution License, which permits unrestricted use, distribution, and reproduction in any medium, provided the original author and source are credited. 
(ascorbic acid) and also on operators handling the device $[5,6,7]$. A well-known interfering factor is the haematocrit value of the blood, which is lower during pregnancy owing to physiological expansion of plasma volume over blood volume. Blood glucose estimations at lower haematocrit can have substantial impact on accuracy of the Glucometer readings. Low haematocrit $<30 \%$ can overestimate the glucose values. Most glucometers were previously calibrated for a fixed haematocrit value of $45 \%$. Newer SMBG devices have employed electrochemistry-based correction algorithms (dynamic electrochemistry) as a possible way to overcome the interfering factors such as haematocrit $[8,9,10]$. Proper education and training to raise the awareness on coding the meters, proper storage conditions for strips, handwashing before use and applying adequate amount of blood is needed, after prescribing the use of the devices.

The significant variation detected among selfmonitoring blood glucose devices has necessitated the development of performance guidelines to assess the validity of the devices [11]. Organizations like American Diabetes Association (ADA), International Standardization Organization (ISO) and Food and Drug Administration (FDA) are involved in development of performance guidelines for SMBG from different manufacturers. Tighter accuracy standards were implemented by ISO (ISO: 15197: 2013) requiring $95 \%$ of the blood glucose measurements to reach an accuracy criteria for values $<100 \mathrm{mg} / \mathrm{dL}$ to be within $\pm 10 \mathrm{mg} / \mathrm{dL}$ and $\pm 20 \%$ for values $\geq 100 \mathrm{mg} / \mathrm{dL}$ [12]. However $15 \%$ variation from true value is considered acceptable by Food and Drug Authority (FDA) for values $>75 \mathrm{mg} / \mathrm{dl}$ and $\pm 15 \mathrm{mg} / \mathrm{dL}$ variation for values $<75 \mathrm{mg} / \mathrm{dL}$ [13] whereas ADA recommends only $5 \%$ variation for all values [14]. However to which extent of analytical accuracy is needed to achieve improved clinical outcomes is in question. Owing to lack of comprehension on accuracy and precision related to SMBG meters available over the counter for lay users make it less reliable for extensive adoption for glycaemia management in diabetes patients [15]. Further the validity and accuracy of different glucometer types vary significantly and at present none of the local authorities carry out pre or post marketing evaluation of these devices in Sri Lanka.

This study aimed to assess the analytical and clinical performance of three different SMBG devices, Device 1, Device 2 and Device 3 which are available over the counter to lay users against a standard laboratory reference method. The accuracy and validity of the readings were evaluated against ISO and ADA approved performance standards for glucometer devices.

\section{Methods}

Randomly selected pregnant mothers who were admitted to antenatal ward, Teaching Hospital Peradeniya, to get blood sugar series test done were recruited on voluntary basis over 3 months' time period from January
2018 to March 2018 (age 21-42 years). At the point of admission for blood sugar series testing, every third mother already diagnosed with diabetes was informed about the study, the samples to be collected, potential outcomes, benefits and risks of the study and informed written consent was obtained from those who were willing to participate. Pregnant women at the Gestational age over 24 weeks were selected. Pregnant women with any bleeding disorder and drips given in arms were excluded. Ethical Clearance was obtained from Ethical Review Committee, Faculty of Medicine, and University of Peradeniya.

Sample size calculation

\begin{tabular}{c|l|}
\cline { 3 - 3 }$(\mathrm{z} 1+\mathrm{Z} 2)^{2} \mathrm{~s}^{2}=\mathrm{n}$ & $\mathrm{n}=$ sample size \\
$\mathrm{d}^{2}$ & $\mathrm{Z} 1=1.96$ for $95 \%$ confidence interval \\
& $\mathrm{Z} 2=0.84$ for $\beta=0.8$ \\
$\mathrm{~s}=0.7$ for standard deviation \\
$\mathrm{d}=0.3 \mathrm{~s}$ for marginal error
\end{tabular}

The calculated minimum sample size was 87 . Around 100 samples were collected in order to increase the accuracy of the analysis.

Three over-the-counter glucometers were evaluated in the study which are designated as Device 1 Device 2 and Device 3 . The three types of new generation glucometer types tested in the study have been used in Teaching Hospital Peradeniya over the past five years. These types are among the brands donated by Ministry of Health, Sri Lanka for clinical use in Hospitals as well. The brands are easily found and are available over the counter to lay users. All were plasma calibrated and contained the automatic calibration or coding feature. However, the electrochemistry methods used by these were undisclosed.

The study comprised of two phases which took place in Teaching Hospital Peradeniya. First, the precision of the glucometers was evaluated by taking repeat measurements using patient samples. Five glucometers for each glucometer type were included. Comparison of laboratory venous blood glucose measurements against capillary whole blood glucometer glucose was carried. Venous blood samples were collected in to tubes containing sodium fluoride-potassium oxalate for blood glucose measurement by laboratory analyzer and EDTA for haematocrit evaluation. Several drops of capillary blood were used to take the glucometer readings from 3 glucometer types. All five glucometers of each type were used in taking the readings. The study testing procedure was carried out by an experienced nursing staff member who was familiar with glucometer functions. To avoid interference from individual variations, all the readings were taken by the same individual. Konelabprime 30I analyzer (Konelab Prime 30I, Thermo Fisher Scientific, USA) was used as the standard reference method for blood glucose estimation in venous plasma samples. The 
haematocrit value at the time of blood collection was also measured with microheamatocrit method.

Statistical analysis was performed using Statistical Software for Social Sciences (SPSS version 20.0) and MATLAB (R2014a, MathWorks Inc., Torrance, CA, USA). The mean and standard deviations was calculated for continuous variables. The mean difference between capillary and venous plasma values were calculated. The accuracy of the glucometer readings was evaluated against ISO and ADA performance standards. Pearson's correlation coefficiency was used to determine the degree of agreement between capillary glucose estimation and venous plasma glucose values of laboratory analysis. Clinical performance of the glucometer devices was further determined by Bland Altman Plots Clarke and surveillance error grids.

\section{Results}

A total 103 diabetic pregnant women were recruited in the study. The mean age of participant women was $31 \pm$ 5 years (age range $21-42$ years). The mean gestational age of the women was 33 weeks. When compared to average venous plasma glucose values, average capillary whole blood glucose values were higher (mean glucose values were $121.5 \mathrm{mg} / \mathrm{dL}$ using the laboratory method, $133.4 \mathrm{mg}$ / $\mathrm{dL}$ in Device $1,125.59 \mathrm{mg} / \mathrm{dL}$ in Device 2 and $137.72 \mathrm{mg} / \mathrm{dL}$ in Device 3). Pearson correlation coefficiency was calculated (Table 1) in order to evaluate the linear relationship between capillary blood glucose values measured with glucometer devices and laboratory venous plasma glucose values. Linear regression model was further used to establish the relationship between the laboratory venous plasma values and capillary blood glucose values measured with different glucometer types (Table 1).

Table 1. Correlation and regression analysis showing degree of agreement between readings of each glucometer type compared against standard laboratory method. (Device $1 ; R^{2}=0.775$, Device 2; $R^{2}=0.733$ and Device 3; $R^{2}=0.762$ )

\begin{tabular}{lccc}
\hline $\begin{array}{c}\text { Glucometer } \\
\text { type }\end{array}$ & $\begin{array}{c}\text { Correlation } \\
\text { coefficiency }\end{array}$ & $R$ square & $\begin{array}{c}\text { Regression } \\
\text { equation }\end{array}$ \\
\hline Device 1 & 0.880 & 0.775 & $\mathrm{Y}=0.880 \mathrm{X}+21$ \\
Device 2 & 0.856 & 0.733 & $\mathrm{Y}=0.856 \mathrm{X}+8.237$ \\
Device 3 & 0.873 & 0.762 & $\mathrm{Y}=0.873 \mathrm{X}+8.8$ \\
\hline
\end{tabular}

The haematocrit range was $26-41 \%$ and range of the glucose concentration was 67-254 mg/dL. Only Device 2 met the ISO performance criteria for glucose values $>100 \mathrm{mg} / \mathrm{dL}$ whereas none met the criteria for values $<100 \mathrm{mg} / \mathrm{dL}$. Meeting American Diabetes Association criteria for accuracy of SMBG devices was not practical to achieve. The haematocrit variation did not have an association with the glucometer error values ( $p>0.05)$.

\section{Bland Altman Plot}

Bland Altman Plot [16] is a mean of assessing the agreement between two quantitative measurements. In contrary to the correlation analysis where relationship between methods are evaluated, Bland Altman analysis estimates the mean difference between 2 methods (bias) and also construct limits for $95 \%$ agreement. All the glucometer devices showed wide limits of $95 \%$ agreement. Device 2 showed the narrowest limits of agreement and minimum constant bias (Figure 1).

\section{Clarke Error Grid}

Clarke error grids were used in determining the clinical accuracy of glucometer devices [17]. The degree of risk for an adverse outcome due to errors in glucose estimation by glucometers were scored for each measured glucose value. The Clarke Error Grid analysis separates the total blood glucose range into five different zones denoted by Zone A to E. Each zone evaluates how glucose variations drive treatment changes in diabetic patients. Both data points in zone A and B are clinically acceptable.

Zone A; Clinically accurate treatment decisions

Zone B; Benign or no treatment error

Zone C; Overcorrection due to falsely increased or decreased glucometer values

Zone D; failure to detect and treat

Zone E; trigger opposite of the correct decision required

The FDA performance standards for glucometers recommends $95 \%$ of the test values to fall within the zone A (also known as allowable total error zone) with $100 \%$ of the values only in zone A and B. All the readings of glucometers evaluated in this study fell within zone A, B and about $1 \%$ in Zone D (Figure 2). Measured values that fell within zone D of all 3 glucometer types lead to failure to detect and treat hypoglycaemia. However, among the different types Device 2 had the maximum readings in zone A $(93.33 \%)$ (Table 2$)$.

Table 2. Percentage values included in each zone of the Clarke Error Grid Analysis

\begin{tabular}{lccccc}
\hline & Zone A & Zone B & Zone C & Zone D & Zone E \\
\hline Device 1 & $73.3 \%$ & $25.5 \%$ & $0 \%$ & $1.1 \%$ & $0 \%$ \\
Device 2 & $83.3 \%$ & $15.6 \%$ & $0 \%$ & $1.1 \%$ & $0 \%$ \\
Device 3 & $62.0 \%$ & $36.8 \%$ & $0 \%$ & $1.1 \%$ & $0 \%$ \\
\hline
\end{tabular}

\section{Surveillance Error Grid (SEG)}

The SEG is a modern method for clinical risk assessment of glucometer errors [18]. The SEG is divided into five zones (extreme, great, moderately, slightly and none) and each zone then again divided into two as hypoglycaemia and hyperglycaemia depending on the risk 
that could be triggered. In surveillance error grid all the data points were within the non-risk zone and slightly lower risk for all glucometer types (both non-risk and slightly risk areas are considered acceptable for glucometer errors).
All the glucometers had data points in the slightly risk zone hypoglyacaemia area indicating clinical error in glucometers with regard to hypoglycemia glucose estimation (Figure 3).

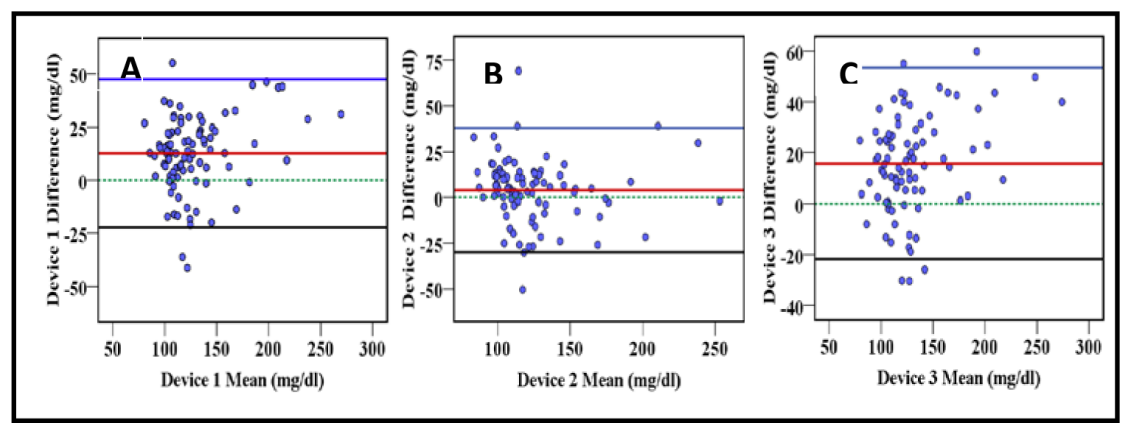

Figure 1. Bland-Altman plots showing the total error between capillary glucose (by Device 1 (A), Device 2 (B) and Device 3 (C)) and venous plasma glucose by laboratory reference method within $95 \%$ limits of agreement. The wide red line parallel to the $X$ axis is the constant bias. The two outer parallel blue and black lines are the values within $95 \%$ limits of agreement (Device 1; 47.36 and -22.29, Device 2; 37.95 and -30.07 and Device 3; 53.35 and -21.76$)$.

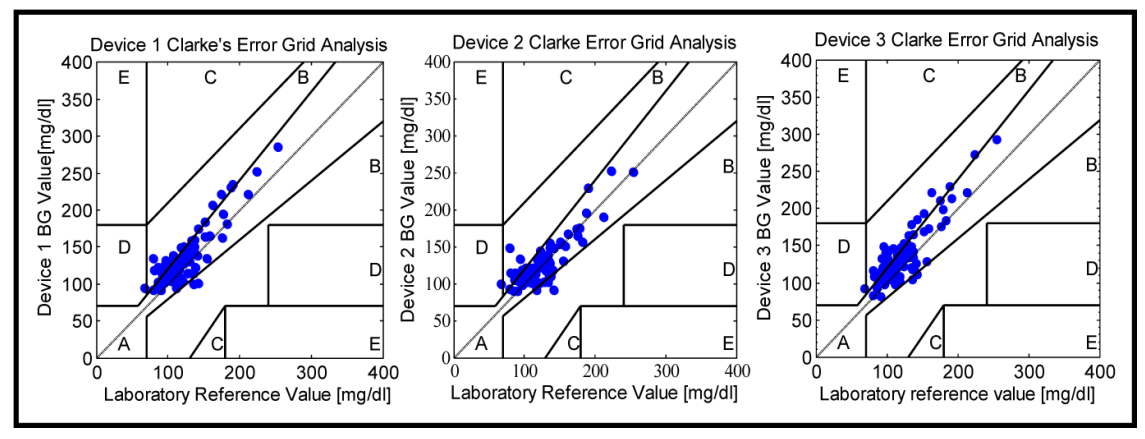

Figure 2. Clarke error grid analysis of showing clinical risk assessment of glucometer errors.

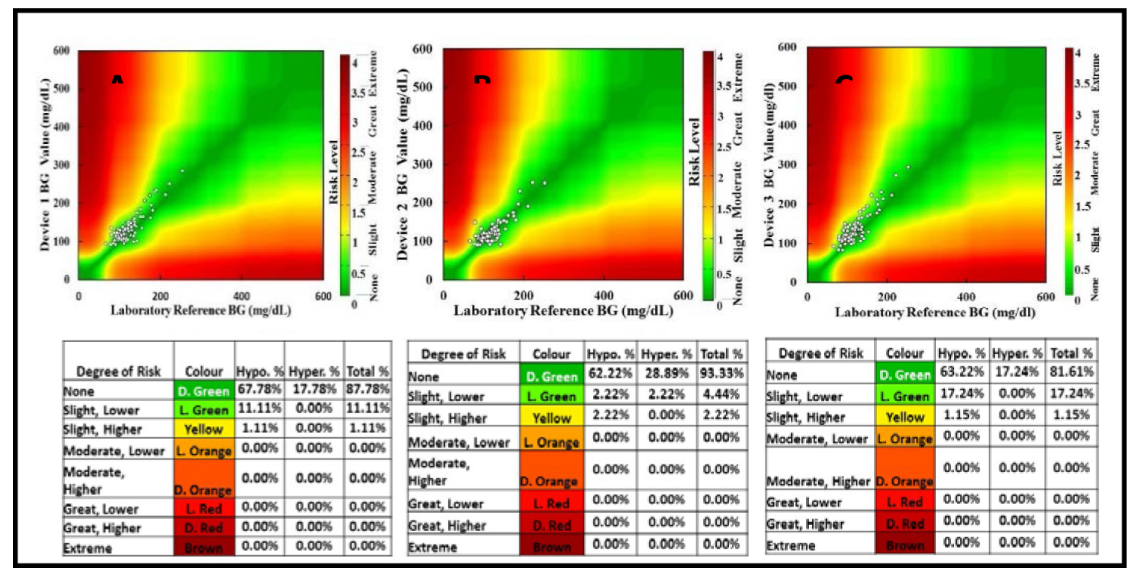

Figure 3. SEG showing risk scores for each glucometer data point against the reference laboratory method (Figure A: Device 1, B: Device 2 and C: Device 3 respectively). 


\section{Discussion}

The glucometer devices had good correlation with the values of standard laboratory method. However previous study by Coyne, Lacour and Meur [19] showed correlation coefficiency of 0.911 for Device 1 and clinical study on Device 3 showed a value of 0.98 [20] which are higher compared to our study. However, those studies had used venous whole blood collected instead of capillary whole blood for which the most glucometers are calibrated. In contrast, our study tested these glucometers in a very practical set-up which created the actual testing environment. The higher mean glucose values observed with glucometer readings can be due to the fact that post prandial capillary blood has higher glucose concentration than venous plasma values [21]. Improper ratio of blood to anticoagulant, inadequate mixing of blood with the anticoagulant, delay in transportation of samples to the laboratory and delay in separation of plasma from whole blood may also cause substantial loss of glucose by glycolysis subsequently resulting in low blood glucose estimation by laboratory method [22]. In terms of clinical consequences of errors of each glucometer type is concerned, most values in no risk zone of Clarke and surveillance error grids was reported by Device 2 (83.3\% and $93.3 \%$ ) whereas almost all readings (99\%) of other glucometer types also fell in acceptable zones, zone A and zone B. However, none of the glucometer types met the performance standards for "total allowable error" for error grid analysis. Pregnant women who requires tighter control should be considered as a special population and proper error grids and percentages of data points that should fall within each zone should be constructed. Among the glucometer types, Device 2 had the best performance in terms of analytical and clinical accuracy. However, none of the glucometers met the ISO criteria for performance standards for values $<100 \mathrm{mg} / \mathrm{dL}$. According to literature most point of care devices are less capable of detecting hypoglycemia [23]. More data should be collected from diabetic pregnant women with blood glucose values $<100 \mathrm{mg} / \mathrm{dL}$ to confirm the finding. Although these devices may not be very accurate to be used in diagnostic purposes, still there is a room for them to be used in glycaemia management as clinical consequences are least affected by minor errors detected. Therefore, a prescription of validated glucometer devices can be put in place for diabetes management during pregnancy as a low cost and easy approach. The decision related to frequency and different time schedules for use of SMBG in order to monitor actual blood glucose values depends on the individual patient and also on the clinician. That information can empower the patients in modifying their lifestyle and also in participating actively in therapeutic management of glycaemia to reduce adverse perinatal outcomes. A clinical trial recruiting more patients for glycaemia management with glucometers can comprehend the validity of these devices for glyacaemia management. Proper training and education should be provided to lay users prior to initiating use of blood glucose monitoring systems owing to confounding nature of factors affecting the glucometer accuracy [24].

However, it can be concluded that, the over the counter SMBG monitoring systems are encountering problems in terms of accuracy and validity. Hence, presales and post-sales assessment of the validity and risk associated with SMBG monitoring systems should be conducted. Glucometer readings indicating hypoglycemia is not reliable and clinicians should exercise caution in making clinical decisions based on glucometer derived glucose values when handling diabetes patients in pregnancy.

\section{Acknowledgments}

We thank Dr. Suranga Kodithuwakku, Department of Animal Science, Faculty of Agriculture, University of Peradeniya for providing the microhaematocrit centrifuge for Haematocrit evaluation.

\section{Conflict of interests}

There are no conflicts of interests.

\section{References}

1. Global report on diabetes. World Health Organization, 2016.

2. Reece, Albert E. The Fetal and Maternal Consequences of Gestational Diabetes Mellitus. J Matern Fetal Neonatal Med 2010; 23(3): 199-203.

3. Standards of Medical Care in Diabetes. American Diabetes Association, 2013.

4. Bettina I, Delamou A, et al. Detection and Management of Diabetes during Pregnancy in Low Resource Settings: Insights into Past and Present Clinical Practices. J Diabetes Res 2016; 2016: 1-14.

5. Tonyushkina, Ksenia, and Nichols, JH. Glucose Meters: A Review of Technical Challenges to Obtaining Accurate Results. J Diabetes Sci Technol 2009; 3(4): 971-80.

6. Ginsberg, Barry H. Factors Affecting Blood Glucose Monitoring: Sources of Errors in Measurement. J Diabetes Sci Technol 2009; 3(4): 903-13.

7. Negrato, Antonio, C and Zajdenverg, L. Self-Monitoring of Blood Glucose during Pregnancy: Indications and Limitations. Diabetol Metab Syndr 2012; 4(1) 54.

8. Demircik F, Hengesbach C, Flacke F, Sieber J, Forst, T. Blood Glucose Meters Employing Dynamic Electrochemistry Are Stable against Hematocrit Interference in a Laboratory Setting. Diabetes Sci Technol 2013; 7(6): 1530-37.

9. Ramljak, Sanja, Lock JP, et al. Hematocrit Interference of Blood Glucose Meters for Patient Self-Measurement. J Diabetes Sci Technol 2013; 7(1): 179-89.

10. Schrot, RJ, Patel KT, Foulis P. Evaluation of Inaccuracies in the Measurement of Glycemia in the Laboratory, by 
Glucose Meters, and through Measurement of Hemoglobin A1C. Clin Diabetes 2007; 25(2): 43-9.

11. Boren, Austin S, and Clarke WL. Analytical and Clinical Performance of Blood Glucose Monitors. J Diabetes Sci Technol 2010; 4(1): 84-97.

12. ISO 15197-2013, in vitro diagnostic test systems - requirements for blood-glucose monitoring systems for self-testing in managing diabetes mellitus. International Organization for Standardization, 2013.

13. Food and Drug Administration. "Blood Glucose Monitoring Test Systems for Prescription Point-of-Care Use-Draft Guidance for Industry and Food and Drug Administration Staff ". Food and Drug Administration, 2014.

14. Walsh J, Roberts R, Robert A, Vigersky, and Schwartz F. New Criteria for Assessing the Accuracy of Blood Glucose Monitors Meeting, October 28, 2011. J Diabetes Sci Techno 2012; 6(2): 466-74.

15. Joakim H. Glucose Measurement: Time for a Gold Standard. J Diabetes Sci Technol 2007; 1(2): 169-72.

16. Bland JM, Altman DG. Statistical methods for assessing agreement between two methods of clinical measurement. Lancet 1986; 1: 307-10.

17. Clarke WMD, Original Clarke Errr Grid Analysis. Diabetes Technol Ther 2005; 7(5): 776-9.
18. Klonoff D, Lias C, Vigersky R, Clarke W, et al. Error Grid Panel: The Surveillance Error Grid. J Diabetes Sci Technol Electronically published on 13 June 2014.

19. Coyne S, Lacour B, Meur HC. Evaluation of Optium Xceed (Abbott) and One Touch Ultra (Lifescan) glucose meters. Ann Biol Clin 2008; 66(3): 249-54.

20. Evaluation of the Device 3 Neo Blood Glucose and Ketone Monitoring System $2013 \mathrm{https}: / /$ freestylediabetes.co.uk/ images/uploads/documents/White_Paper __Clinical_FreeStyle_Optium_Neo.pdf (Acessed on July 10, 2018).

21. Yang C, Chang C and Lin J. A Comparison between Venous and Finger-Prick Blood Sampling on Values of Blood Glucose. IPCBEE 2012; 39: 206-10.

22. Gupta, Shalini, Kaur H. Inhibition of Glycolysis for Glucose Estimation in Plasma?: Recent Guidelines and Their Implications. Ind J Clin Biochem 2014; 29: 262-4.

23. Sonmez A, Kilic S, Taper S, Taslipinar A. The Accuracy of Home Glucose Meters in Hypoglycemia. Diabetes Technol Ther 2010; 12: 619-26.

24. Klonoff DC. Point-of-Care Blood Glucose Meter Accuracy in the Hospital Setting. Diabetes Spectrum 2014; 27(3): 174-9. 